\title{
PULMÓN REUMATOIDE
}

José Ignacio Hernández Cruz MD*, Publio Giovanny Saavedra Ramírez MD**

Se describe el caso de un paciente de 62 años, atendido en el Servicio de Medicina Interna del Hospital de San José, con apoyo de los Servicios de Neumología y Cuidados Intensivos. Residente y procedente de la ciudad de Bogotá; ingresó por tos, dificultad respiratoria y fiebre de tres días de evolución, acompañadas de astenia, adinamia, expectoración mucosa y disnea que progresó rápidamente desde la clase funcional I a la III. Como antecedente de importancia, se incluye diagnóstico de artritis reumatoidea (AR) dos años antes, en tratamiento con metotrexate $12,5 \mathrm{mg} / \mathrm{semanales}$, prednisolona $10 \mathrm{mg} /$ día, cloroquina $250 \mathrm{mg} /$ día, ácido fólico $1 \mathrm{mg} /$ día y diclofenaco $50 \mathrm{mg}$ dos veces al día, con control adecuado de sus síntomas. No hay más datos de importancia.

Al examen físico se encontró paciente en regular estado general, con dificultad respiratoria por taquipnea; a la auscultación pulmonar se escuchaban estertores gruesos en la base del hemitórax derecho y finos en la base del izquierdo, sin signos de consolidación o de derrame pleural.

Se hizo una impresión inicial diagnóstica de:

1. Neumonía adquirida en la comunidad en paciente inmunosuprimido.

2. Toxicidad pulmonar por metotrexate.

3. Síndrome de dificultad respiratoria aguda asociado.

4. AR en tratamiento.

Los exámenes paraclínicos de ingreso reportaron: hematócrito $37 \%$, blancos $10.020 \mathrm{cel} / \mathrm{ml}$, neutrófilos $9.590 / \mathrm{ml}$, linfocitos $720 / \mathrm{ml}$, plaquetas $229.000 / \mathrm{ml}$ VSG: $57 \mathrm{~mm} / \mathrm{h}$, creatinina $1.46 \mathrm{mg} / \mathrm{dl}$, nitrógeno ureico $31 \mathrm{mg} / \mathrm{dl}$, glucemia $259 \mathrm{mg} / \mathrm{dl}, \mathrm{Na}^{+} 133 \mathrm{mEq} / \mathrm{L}, \mathrm{K}^{+} 4,7$ $\mathrm{mEq} / \mathrm{L}$, deshidrogenasa láctica $664 \mathrm{mg} / \mathrm{dl}$, proteína C

\footnotetext{
* Internista Cardiólogo, Jefe del Servicio de Medicina Interna Hospital de San José. Bogotá, Colombia

** Residente 3er. año Medicina Interna.
}

reactiva $31,35 \mathrm{mg} / \mathrm{dl}$ y gases arteriales tomados con una $\mathrm{FiO}_{2}$ del 35\% que mostraban $\mathrm{pH} 7,40 ; \mathrm{pCO}_{2} 24 \mathrm{~mm} /$ $\mathrm{Hg}, \mathrm{pO}_{2} 50 \mathrm{~mm} / \mathrm{Hg}, \mathrm{HCO}_{3} 15 \mathrm{mMol} / \mathrm{L}$ y saturación $84 \%$.

Las aminotransferasas estaban levemente aumentadas: AST 46 y ALT 51. La radiografía inicial de tórax (Figura 1) mostró infiltrado intersticial retículonodular y peribroncovascular de distribución difusa en ambas bases pulmonares, engrosamiento pleural en los vértices pulmonares y signos de disminución de volúmenes pulmonares. En la Figura 2 se observa el rápido compromiso pulmonar experimentado por este enfermo.

Debido a la sospecha de infección pulmonar se inició manejo antibiótico con ceftriaxone, y ante la posible presencia de gérmenes oportunistas como $P$. carinii, se decide adicionar trimetoprim-sulfametoxazol. El Gram de esputo reportó flora mixta y el seriado del mismo fue negativo para bacilos ácido-alcohol resistentes. Se solicitó coloración de Gomory (metenamina de plata), la cual fue reportada como negativa.

El paciente presentó rápido deterioro desde el punto de vista respiratorio, con disnea de reposo, cianosis central y periférica, y compromiso severo de los índices de oxigenación en los gases arteriales,

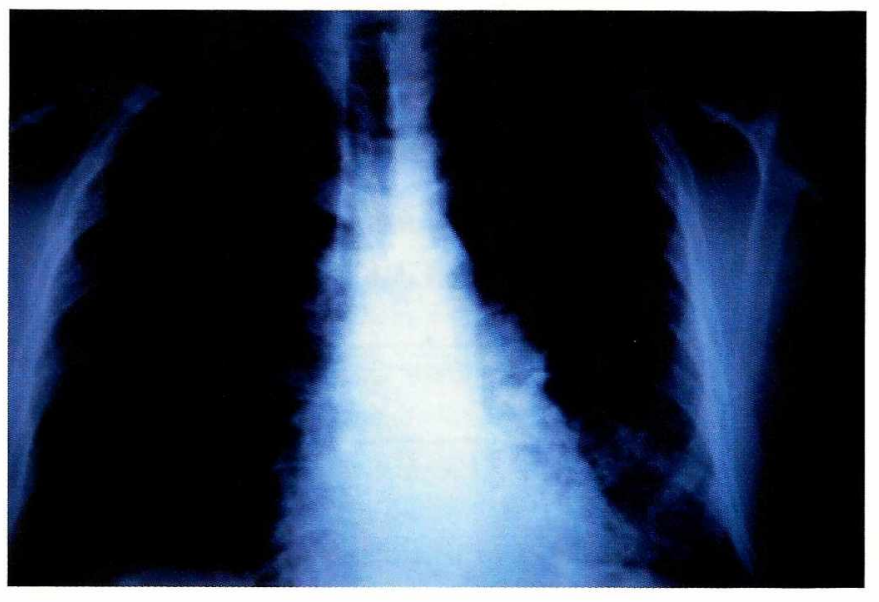

Figura I. Radiografía de tórax que muestra infiltrado intersticial reticulonodular y peribroncovascular de distribución difusa en ambas bases pulmonares. 


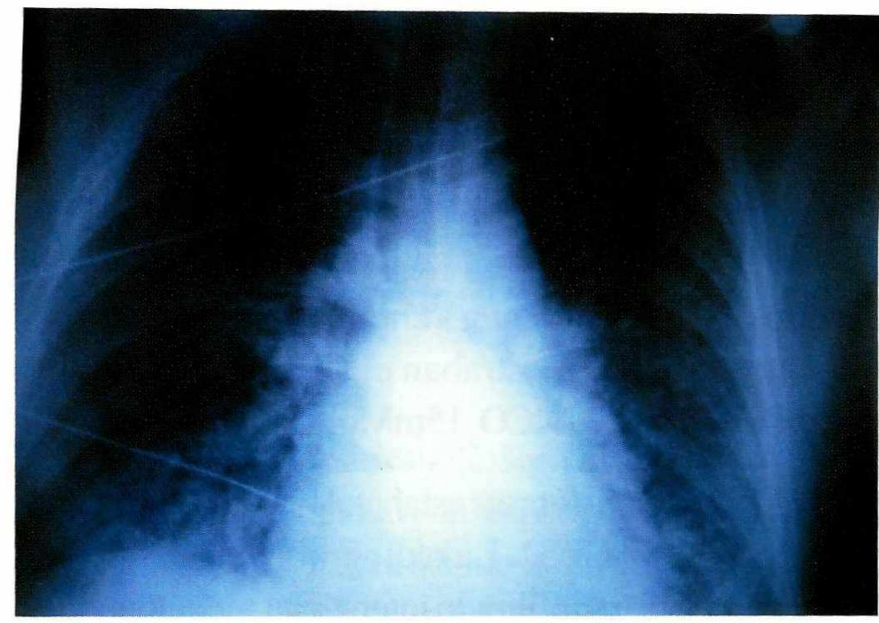

Figura 2. La radiografía evidencia rápido compromiso pulmonar.

dado por una relación $\mathrm{PaO}_{2} / \mathrm{FiO}_{2}$ de 100 . Fue trasladado a la UCI, donde se inició ventilación no invasora, pero debido al deterioro progresivo se realizó intubación orotraqueal con soporte ventilatorio mecánico. Al tercer día de hospitalización, el Servicio de Neumología practicó fibrobroncoscopia para lavado broncoalveolar (LBA) y biopsia transbronquial, con el ánimo de descartar un posible proceso infeccioso. El estudio microbiológico fue negativo, mientras que la celularidad del LBA mostró polinucleares $81 \%$, mononucleares $10 \%$, células en cepillo $2 \%$ y macrófagos alveolares $7 \%$.

Ese mismo día se obtuvieron datos de $\mathrm{C} 3-\mathrm{C} 4$ dentro de límites normales, anti-DNA negativo y factor reumatoideo muy positivo: $95 \mathrm{UI} / \mathrm{ml}$ (V.N: 40). La prueba de Elisa para VIH se informó negativa. Se so- licitó tomografía computarizada (TAC) de tórax de alta resolución, que mostró opacidad en vidrio esmerilado en ambos parénquimas pulmonares, consolidación de los segmentos basales izquierdos y del lóbulo inferior derecho en su totalidad, dilatación bronquial moderada y nódulos pequeños en ambos hemitórax (Figu$\operatorname{ras} 3,4$ y 5 ).

Debido a la negatividad de todos los estudios microbiológicos, incluyendo cuatro hemocultivos, y al sospechar que el compromiso pulmonar tenía origen inmunológico por su enfermedad de base, se decidió iniciar bolos de metilprednisolona con dosis total de $3 \mathrm{~g}$, con lo cual se obtuvo una mejoría transitoria, pero con posterior deterioro y empeoramiento del estado clínico del paciente, con dependencia del soporte ventilatorio mecánico y requerimiento progresivo de mayores PEEP y presión de soporte.

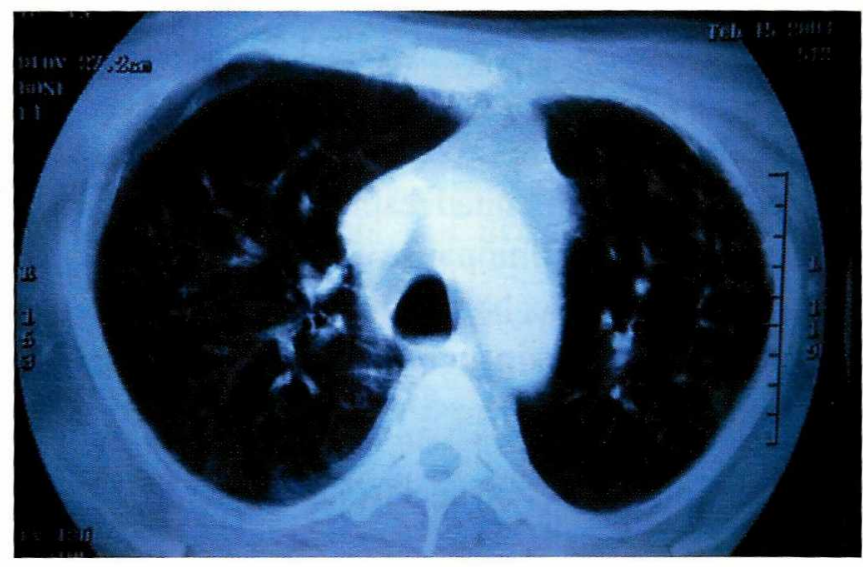

Figura 3. La radiografía de tórax evidencia opacidad en vidrio esmerilado de ambos parénquimas pulmonares.

\begin{tabular}{|c|c|c|c|c|c|}
\hline Pleura & Nódulos & Intersticio & Vasos & Vías aéreas & Varios \\
\hline Pleuritis & Necrobióticos & $\begin{array}{l}\text { Fibrosis } \\
\text { Pulmonar }\end{array}$ & Vasculitis & Obstrucción & $\begin{array}{l}\text { Toxicidad } \\
\text { medicamentosa }\end{array}$ \\
\hline Derrame & S. de Caplan & & $\begin{array}{l}\text { Hipertensión } \\
\text { pulmonar }\end{array}$ & B.O.O.P & Infección \\
\hline Neumotórax & $\begin{array}{l}\text { Nodulosis } \\
\text { reumatoidea }\end{array}$ & & & $\begin{array}{l}\text { Bronquiolitis } \\
\text { obliterante }\end{array}$ & Amiloidosis \\
\hline Empiema & & & & $\begin{array}{l}\text { Bronqui- } \\
\text { ectasias }\end{array}$ & $\begin{array}{l}\text { Enf. Fibro- } \\
\text { bullosa }\end{array}$ \\
\hline $\begin{array}{l}\text { Fístula bronco- } \\
\text { pleural }\end{array}$ & & & & $\begin{array}{l}\text { Artritis crico- } \\
\text { aritenoide }\end{array}$ & \\
\hline
\end{tabular}




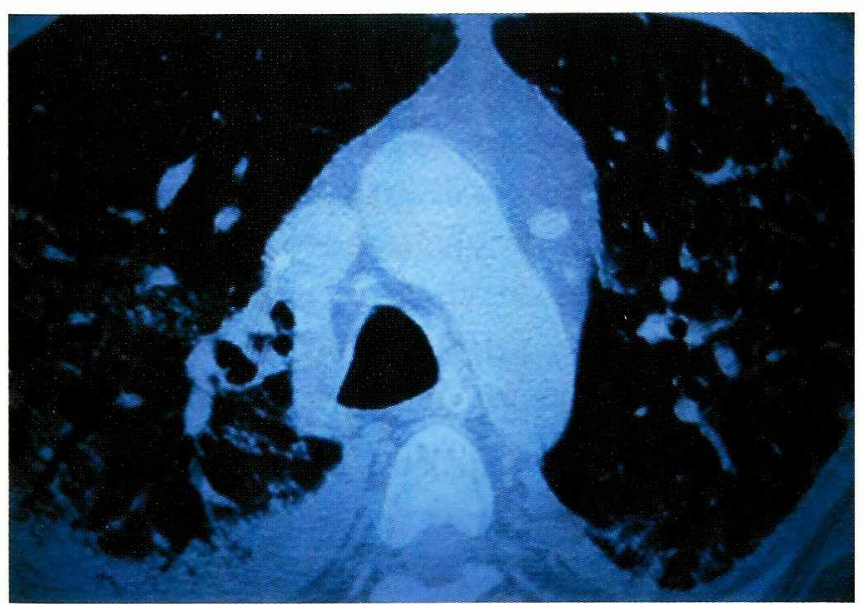

Figura 4. La radiografía de tórax evidencia consolidación de los segmentos basales bilaterales.

El informe patológico del tejido transbronquial muestra cambios de fibrosis intersticial. Se realiza biopsia pulmonar abierta el día 18 de su estancia hospitalaria, la cual reporta neumonía intersticial usual con extensas áreas de fibrosis terminal.

El paciente continúa con alteración grave de su función respiratoria. Presenta picos febriles y se diagnostica neumonía asociada al ventilador, con hemocultivos positivos para Acinetobacter baumanni multirresistente. Aparece disfunción orgánica múltiple asociada a su proceso infeccioso y fallece en el día 22 de su estancia hospitalaria.

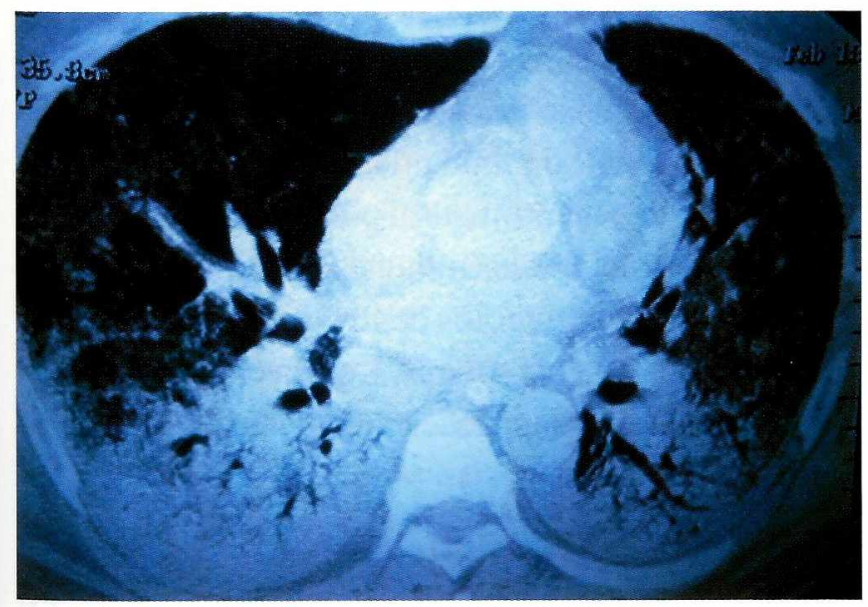

Figura 5. La radiografía de tórax evidencia la misma consolidación bibasal con dilatación bronquial moderada y nódulos pequeños bilaterales.

\section{Discusión}

La AR es una enfermedad inflamatoria, sistémica y de curso crónico, de causa desconocida, con un patrón característico de afección de las diartrosis. El trastorno articular suele estar acompañado de factor reumatoide y compromiso extraarticular variable; la artritis es la principal manifestación ${ }^{1}$.

Puede presentarse a cualquier edad, pero la mayoría de los casos se observan entre la tercera y quinta década de la vida. Predomina en el sexo femenino 2 a 3:1. La incidencia se encuentra en promedio entre $20 \mathrm{y}$ 50 casos nuevos por 100.000 habitantes/ año. La prevalencia se estima en $1 \%$ de la población mundial ${ }^{2}$.

\section{Manifestaciones pulmonares}

LaAR es una enfermedad generalizada con frecuentes manifestaciones extraarticulares, incidencia acumulativa y puede afectar casi a cualquier órgano. El espectro de la enfermedad pulmonar asociada es muy amplio (Tabla 1). Además, las complicaciones pulmonares relacionadas con el tratamiento, entre ellas la infección, merecen ser tenidas en cuenta en la evaluación y manejo de estos pacientes.

\section{Enfermedad pulmonar intersticial}

La enfermedad pulmonar intersticial (EPI) es la manifestación pulmonar más común de la $\mathrm{AR}$. En muchos casos es clínicamente silente y se identifica en forma casual por anormalidades radiológicas, pruebas fisiológicas pulmonares o por cambios en el perfil celular del LBA. Según la modalidad diagnóstica utilizada para detectar la enfermedad, las tasas de prevalencia de la EPI en la AR son reportadas dentro de un amplio rango de variación. En general, la EPI es más frecuente en hombres que en mujeres, presenta títulos altos de factor reumatoideo y se acompaña de manifestaciones articulares más severas.

Desde el punto de vista radiológico, los cambios observados en la EPI de la AR son indistinguibles de aquellos de la fibrosis pulmonar idiopática o de la EPI asociada con otras enfermedades del tejido conectivo. El hallazgo más común es un infiltrado intersticial bibasal bilateral, que a menudo es asimétrico. Temprano en el 
curso de la enfermedad, este cambio puede aparecer como infiltrado de ocupación alveolar en parches. La progresión de la enfermedad resulta en un patrón más retículonodular. La radiografía convencional de tórax es un método poco sensible para identificar la EPI, dando una tasa de prevalencia del 2 al 6\%. La TAC de tórax de alta resolución es un método más sensible para detectar los cambios intersticiales fibrosantes en la EPI de la AR. La prevalencia de los cambios identificados por dicho método oscilan entre 10 a 47\%. El TAC de tórax de alta resolución puede además identificar otras anormalidades asociadas, como son bronquiectasias, nódulos pulmonares, linfadenopatía y enfermedad pleural.

Las anormalidades fisiológicas son comunes en la $\mathrm{AR}$. Se han reportado alteraciones sugestivas de EPI en 22 a $40 \%$ de los pacientes. Los cambios más frecuentes son paralelos a aquellos vistos en la EPI de otra etiología, e incluyen disminución de los volúmenes pulmonares, reducción en la elasticidad pulmonar y alteraciones en la capacidad de difusión del monóxido de carbono. Muchos pacientes con artritis y radiografía normal de tórax pueden tener alteraciones en las pruebas de función pulmonar, que, en ocasiones, muestran evidencia de obstrucción al flujo de aire, lo cual puede reflejar la presencia de otras manifestaciones pulmonares de la artritis, incluyendo bronquiectasias, bronquiolitis obliterante, obstrucción crónica de la vía aérea o artritis de los cartílagos cricoaritenoides.

El LBA ha sido muy utilizado como herramienta para el estudio de las EPI. Los informes de la composición del LBA en la EPI asociada con AR han demostrado variación considerable; se ven en el 50 a $60 \%$ de los casos. En aquellos sin evidencia de EPI, tienden hacia un perfil celular del LBA normal, pero también pueden mostrar alveolitis linfocítica. En contraste, pacientes con AR y evidencia clínica de EPI suelen demostrar una alveolitis neutrofílica y aumento del número absoluto de macrófagos. Es de anotar que patrones similares se encuentran en la fibrosis pulmonar idiopática. Cabe recordar que la linfocitosis en el LBA se asocia con mejor pronóstico y buena respuesta al tratamiento con corticoides, mientras que la alveolitis neutrofílica indica un peor pronóstico y mala respuesta a los esteroides. Los macrófagos alveolares recuperados de pacientes con AR con o sin EPI detectable clínicamente, parecen encontrarse activados, pues se demuestran grandes cantidades de anión superóxido, fibronectina, factor quimiotáctico activador de los neutrófilos y factor de necrosis tumoral. Estos hallazgos se han descrito en pacientes con recuento celular diferencial normal en el LBA, lo cual sugiere que la activación de los macrófagos alveolares puede preceder al reclutamiento de células inflamatorias en el intersticio pulmonar.

En un estudio reciente se examinaron 36 pacientes con AR de menos de dos años de duración, mediante radiografía de tórax, TAC de alta resolución, pruebas de función pulmonar y LBA. En promedio, 58\% de estos pacientes mostró anormalidades sugestivas de EPI en por lo menos uno de estos exámenes. Individualmente, en el $6 \%$ fueron anormales las radiografías de tórax, $33 \%$ en la TAC de tórax, $22 \%$ en las pruebas de función pulmonar y $52 \%$ en el LBA. Estos resultados resaltan la variación con la cual la prevalencia de la EPI en la AR puede ser identificada, aun en un mismo grupo de pacientes. Además, estos autores identificaron una EPI clínicamente significativa, definida por la presencia de signos y síntomas, anomalías radiográficas y fisiología pulmonar restrictiva, o hallazgos anormales del LBA, en $14 \%$ de sus pacientes, lo cual enfatiza el hecho que la EPI es una complicación frecuente de los pacientes con AR.

\section{Patogénesis}

No se conocen con exactitud los mecanismos responsables del desarrollo de la EPI en pacientes con AR. Se asume que los procesos mediados inmunológicamente en la AR son fundamentales en el proceso inflamatorio que compromete al pulmón. Es posible que el depósito de complejos inmunes en el intersticio y en las paredes de los alvéolos contribuya a la activación de los macrófagos alveolares. Los mecanismos de hipersensibilidad celular pueden desempeñar un papel importante en la patogénesis.

Debido a que la EPI de la AR se asocia con la presencia de factor reumatoideo, se ha considerado la potencial patogenicidad del factor reumatoideo IgM por sí mismo. En los pacientes con AR se han podido demostrar, extensos depósitos de dicho factor en las pa- 
redes alveolares y en las arteriolas pulmonares. En menor grado, se hallan IgG e IgA. Sin embargo, el factor reumatoideo IgM no parece ser el principal mediador del daño pulmonar en la AR.

\section{Patología}

Desde el punto de vista histológico, los cambios vistos en la EPI asociada con la AR son paralelos a aquellos de la fibrosis pulmonar idiopática. En las etapas tempranas predomina un infiltrado intersticial compuesto en esencia por linfocitos, células plasmáticas e histiocitos. A medida que la enfermedad progresa, se hace menos pronunciado y se reemplaza por tejido fibroso. Los casos severos llegan a pulmón en estadio terminal o "panal de abejas". Sin embargo, todos estos cambios no son específicos de la afección. Los estudios de inmunofluorescencia pueden demostrar depósitos de inmunoglobulinas, particularmente de $\operatorname{IgM}$, en las paredes alveolares y arteriolares. El infiltrado linfocítico intersticial de la AR puede formar agregados nodulares focales con centros germinales, una característica que no se ve en la fibrosis pulmonar idiopática. El hallazgo de nódulos necrobióticos suele señalar la presencia de AR. Además, existen algunas otras características como la fibrosis pleural o la bronquiolitis obliterante con neumonía organizada, que ayudan a diferenciar la EPI de la AR con otras enfermedades pulmonares intersticiales.

\section{Manifestaciones clínicas}

Los síntomas de la EPI ocurren después del inicio de la AR en la mayoría de los pacientes, de manera que el diagnóstico de AR ya está establecido. Sin embargo, las manifestaciones articulares y pulmonares pueden presentarse de manera simultánea, o aparecer primero la enfermedad pulmonar, lo cual sucede en $20 \%$ de los casos. Por lo general, las alteraciones articulares y pulmonares se desarrollan en un lapso de cinco años. La severidad no siempre es igual.

En muchos casos los pacientes con EPI y AR son asintomáticos. En otros, la disnea y la tos son las manifestaciones más comunes. En casos leves, el examen físico puede ser normal. Cuando aparecen signos, por lo regular consisten en estertores finos bibasales y taquipnea. En $75 \%$ de los pacientes se ven uñas en vidrio de reloj.
En los casos más severos hay falla respiratoria y progresión hasta el pulmón en estado terminal (panal de abejas; 5,8 de los casos). En estas circunstancias pueden surgir signos de hipertensión arterial pulmonar y de insuficiencia cardiaca derecha. En general, la EPI de la AR tiende a tener un curso lento e insidioso. La limitación sintomática severa es poco frecuente.

\section{Tratamiento}

Los corticoides constituyen la terapia inicial. La tasa de respuesta es variable y depende de los parámetros usados para medirla. Los cambios subjetivos en los síntomas y la tolerancia al ejercicio pueden o no ser paralelos con la mejoría radiológica, los hallazgos en las pruebas de función pulmonar, el LBA y otros procedimientos de valoración.

La decisión para iniciar el tratamiento se basa en la combinación de hallazgos clínicos, radiológicos y fisiológicos; se recomienda biopsia y estudio histológico. $\mathrm{La}$ dosis inicial de corticoides varía entre 0,5 a $1 \mathrm{mg} / \mathrm{kg} /$ día de prednisona. La disminución se decide por la mejoría objetiva de la tolerancia al ejercicio, las anomalías radiológicas y las pruebas de función pulmonar. Cuando no es posible prescribir esteroides o estos han fallado, se utilizan otros fármacos inmunosupresores como el metotrexate, azatioprina o ciclofosfamida, los cuales se consideran efectivos en casos aislados. Hasta la fecha no se han realizado grandes estudios sobre la respuesta a estos agentes.

\section{Toxicidad pulmonar inducida por metotrexate}

En este paciente, uno de los diagnósticos diferenciales iniciales fue precisamente la toxicidad pulmonar inducida por metotrexate, la cual se puede manifestar de diferentes formas: neumonitis intersticial aguda, fibrosis intersticial, edema pulmonar no cardiogénico, pleuritis con derrame y nódulos pulmonares.

La dificultad para determinar si un paciente con AR tiene una fibrosis intersticial inducida por medicamentos o asociada con la enfermedad reumática de base, es una pregunta clínica importante que muchas veces no puede contestarse. El curso clínico de un paciente típico con toxicidad pulmonar inducida por metotrexate es el de una enfermedad subaguda, caracterizada por tos 
no productiva, fiebre y diagnóstico de infección respiratoria alta. Si no se da tratamiento, la enfermedad puede progresar hasta poner en peligro la vida, necesitando soporte ventilatorio mecánico y altas dosis de corticosteroides. No existen hallazgos clínicos específicos de esta afección. El tiempo transcurrido entre el inicio del metotrexate y el desarrollo del cuadro clínico es muy variable: entre una y 480 semanas. También es variable la dosis acumulada: desde 7,5 hasta $3.600 \mathrm{mg}$. Infortunadamente no existen pruebas de laboratorio específicas para el diagnóstico preciso de esta condición y las que se usan tienen como finalidad excluir otras patologías, como por ejemplo infecciones oportunistas que ocurren con el uso de esta medicación. Ni la clínica, los estudios radiológicos ni las pruebas de función pulmonar, incluso el BAL, pueden proveer información suficiente para hacer el diagnóstico preciso.

Histológicamente son tres las características de la toxicidad pulmonar inducida por el metotrexate: neumonitis intersticial, bronquiolitis y formación de células gigantes. Los linfocitos son las células dominantes en la inflamación intersticial de la neumonitis inducida por el metotrexate, con ocasionales polimorfonucleares. También se ha visto la formación de granulomas con células gigantes, edema alveolar y exudados que pueden progresar hasta el daño alveolar difuso.

En cuanto al manejo, se deben realizar tres acciones: suspender el medicamento, medidas generales de soporte y tratamiento con corticoides. Con ello, la mayoría de los pacientes con enfermedad pulmonar inducida por metotrexate experimenta una recuperación completa de la función pulmonar.

En conclusión, hemos presentado un caso de fibrosis pulmonar asociada con $\mathrm{AR}$, que hace su aparición clínica a los dos años del inicio de las manifestaciones articulares. La evolución fue rápida hacia fibrosis terminal y pulmón en "panal de abejas", hasta llegar a la insuficiencia respiratoria. Se analiza la correlación clínica, radiológica, paraclínica (LBA) y patológica. Durante el proceso diagnóstico se descartaron patologías infecciosas oportunistas o afecciones inmunológicas diferentes a la AR, como causa probable de la enfermedad pulmonar de este paciente. Los hallazgos paraclínicos y de patología, así como el desenlace fatal, no soportan el diagnóstico de toxicidad por metotrexate.

\section{Lecturas recomendadas}

1. Tanoue $\mathbf{L}$. Thoracic manifestations of the systemic autoinmune diseases. Pulmonary manifestations of rheumatoid arthritis. Clin Chest Med 1998; 19: 667-685.

2. Barkhuizen A, Campbell S. Pulmonary involvement in common rheumatologic diseases in the elderly. Imm Aller Clin North Am 1997; 17: 727-744.

3. Cannon G. Methotrexate pulmonary toxicity. Rheum Clin North Am 1997; 23: 917-937.

4. American Thoracic Society / European Respiratory Society International Multidisciplinary Consensus Classification of the Idiopathic Intersticial Pneumonias. Am J Respir Crit Care Med 165: 277-304, 2002.

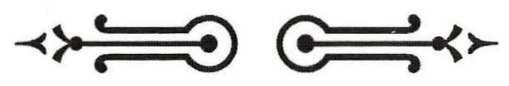

